\title{
ANALYSIS OF LUMPED PARAMETER MODELS FOR BLOOD FLOW SIMULATIONS AND THEIR RELATION WITH 1D MODELS
}

\author{
Vuk Milišić $^{1}$ AND Alfio QuARteroni ${ }^{1,2}$
}

\begin{abstract}
This paper provides new results of consistence and convergence of the lumped parameters (ODE models) toward one-dimensional (hyperbolic or parabolic) models for blood flow. Indeed, lumped parameter models (exploiting the electric circuit analogy for the circulatory system) are shown to discretize continuous 1D models at first order in space. We derive the complete set of equations useful for the blood flow networks, new schemes for electric circuit analogy, the stability criteria that guarantee the convergence, and the energy estimates of the limit 1D equations.
\end{abstract}

Mathematics Subject Classification. 35L50, 35M20, 47H10, 65L05, 76Z05.

Received: February 6, 2004. Revised: March 24, 2004.

\section{INTRODUCTION}

The cardiovascular system can be regarded as a wide hydraulic network under the action of a pulsatile pump. Different behavior can be observed at various locations of the closed loop. For instance in the arterial tree, the wave propagation is of greater influence while in the capillary bed, the flow is almost steady, putting into evidence the lumped character of the system. On the other hand, local phenomena, like anastomosis for instance, create flow perturbations upwardly and downwardly. This shows the interdependency of different scales of the system, leading to a multiscale approach.

Some simplified models take into account the previous properties of the cardiovascular system. For instance the Windkessel and similar lumped models are often used to represent blood flow and pressure in the arterial system $[1,12,16,22]$. These lumped models can be derived from electrical circuit analogies where current represents arterial blood flow-rate and voltage represents arterial pressure. Resistances represent arterial and peripheral resistance that occur as a result of viscous dissipation inside the vessels, capacitors represent volume compliance of the vessels that allows them to store large amounts of blood, and inductors represent inertia of the blood. Besides being of simple derivation, the main advantage of lumped models is that they are easy to solve since they give rise to simple ordinary differential equations.

On the other hand, one dimensional models of the human arterial system yield partial differential equations expressing the conservation of mass and momentum for inviscid flow (see $[3,9,20]$, and references therein).

Keywords and phrases. Multiscale modelling, parabolic equations, hyperbolic systems, lumped parameters models, blood flow modelling.

${ }^{1}$ Chair of Modelling and Scientific Computing, IACS, École Polytechnique Fédérale de Lausanne, 1015 Lausanne, Switzerland. e-mail: vuk.milisic@epfl.ch

2 MOX, Dipartimento di Matematica, Politecnico di Milano, 20133 Milano, Italy. e-mail: alfio.quarteroni@epfl.ch 
Recently, in [17], it has been shown numerically that the linearization of the one dimensional model around a constant state matches in a very suitable manner the nonlinear system itself, even for very realistic test cases.

Since both lumped parameter and one dimensional models are supposed to model the same physiological behavior, one natural question arises: how can two completely different approaches provide comparative answers?

The first direct derivation of a linearized 1D model from axisymmetric Navier-Stokes equations was carried out in [15]. To solve these equations the authors use the electric analog. Namely, they compute an approximate solution using a standard $R L C$-circuit containing a resistor $R$, an inductor $L$ and a capacitor $C$. In [6], the authors suggest that "the transmission line can be represented to any desired accuracy by a lumped section" with a reference to the book by Mason [11], based on a sinusoidal steady state analysis. But so far, an analytical and numerical evidence of the latter claim is still missing.

In this article, we give this proof. Precisely, for the particular case of blood flow, we prove that lumped networks can be regarded as first order discretizations of one-dimensional linear systems: more precisely, the solutions of lumped networks converge to that of continuous linear 1D systems when $\Delta x$ (the length of a single compartment) and $\Delta t$ (the time step) tend to zero. The convergence is established thanks to Lax-Richtmyer's theorem, after proving stability using Von-Neumann analysis.

First we establish this result for $R L C$ circuits. Starting from the entropy functional associated to the nonlinear one-dimensional hyperbolic system, we derive the energy associated to the linearized one. At the discrete level this energy functional is found to be exactly the instantaneous power dissipated in each compartment of the lumped network. Then the convergence toward a linear hyperbolic system is proven thanks to this energy that "symmetrizes" the scheme.

The standard $R L C$ schemes are shown to be stable (and thus convergent) under a "parabolic" CFL condition which severely penalizes the refinement of the spatial grid. In fact, the diagonalization of the scheme shows a preferential direction of waves propagation. Based on this observation, we propose new 0D schemes that use (less restrictive) hyperbolic CFL condition and do not privilege any direction.

Numerical tests show that the difference between the entropy functional and the linear energy is grid independent and almost negligible for realistic blood flow simulations. Moreover, an approximate order of convergence of the solutions computed by lumped models toward the solutions of the continuous linear 1D system is established, confirming the theoretical prediction.

An asymptotic analysis is carried out for small vessels leading to new nonlinear equations. By linearization we obtain a linear heat equation, which could also be obtained as a degenerate case of the linear 1D model previously introduced. The discretization of the heat equation leads to schemes that are suitable to describe an electric circuit containing only a resistor and a capacitor. Again a stability condition guarantees the convergence toward the linear equation.

While for the electric networks the continuity of physical quantities between different elements is ensured by Kirchhoff's laws, the coupling of continuous PDE models needs more careful treatment of interface conditions. The existence of such couplings will be carried on in a future work [13].

An outline of the paper is as follows. With the aim of enhancing readability, we sketch in Figure 1 the main items of our road map. Dashed lines and boxes outline the most important contributions brought by this paper.

In Section 2, we study the $R L C$ circuits. At first, in Section 2.1, we introduce the $R L C$ network from a novel perspective, by linearizing the cross-sectional 1D approximation of Navier-Stokes equations around a constant state. Then, we use the $R L C$ networks to discretize this linear system. This technique provides exactly the same definition of the $R, L$ and $C$ constants as already found in $[9,15]$. In Section 2.2, we introduce the energy estimates allowing us to prove the convergence result. Analyzing the electric scheme, we provide an alternative way of discretizing the $R L C$ system with different upwinded schemes in Section 2.4. A numerical evidence is displayed in Section 2.5. Then, we perform the same analysis on small vessels leading to the lumped elements associated to the capillary and venous bed (see Sect. 3). Namely, in Section 3.1, we derive an incomplete one-dimensional hyperbolic system which is indeed a scalar parabolic equation for blood pressure. 


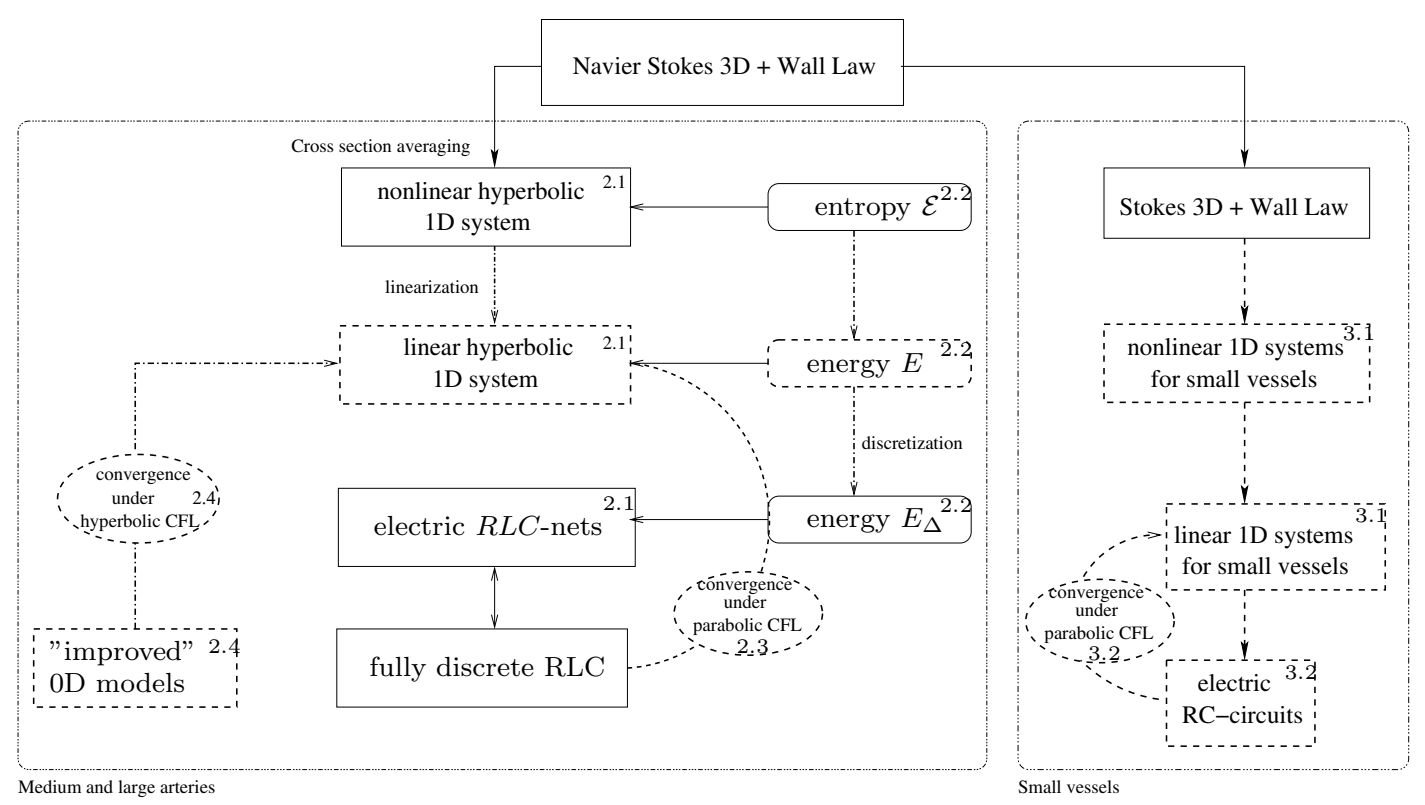

FIGURE 1. A sketch of the hierarchical multiscale models addressed in this paper and the main theoretical results; numbering refers to subsections.

Then in Section 3.2, by space integration we associate a OD ODE system which models a RC electric circuit. At this stage we have built a complete hierarchy of nonlinear-linear 1D systems and lumped parameters for blood flow.

\section{From Linear CONSERVATION LAWS to RLC NETWORKS AND VICE-VERSA}

\subsection{Deriving the basic $\mathcal{L}$-circuit equations}

A basic description of the $R L C$ network as an approximation of the nonlinear conservation law for blood flow can be found in [9]. The authors use various considerations such as linearization arguments and physical assumptions. Here we introduce the $R L C$ network by following a new approach.

Starting from a simplified version of the Navier-Stokes equations in the axisymmetric form and integrating these equations over each cross-section $A(x, t)$ of the vessel (see $[2,3,18]$ and references therein) we obtain the following set of $1 \mathrm{D}$ equations, for $0<x<l$ ( $l$ being the vessel length) and all $t>0$ :

$$
\left\{\begin{array}{l}
\partial_{t} A+\partial_{x} Q=0, \\
\partial_{t} Q+\partial_{x}\left(\frac{\alpha Q^{2}}{A}\right)+\frac{A}{\rho} \partial_{x} P=-K_{r} \frac{Q}{A},
\end{array}\right.
$$

where $\alpha$ (the momentum-flux correction coefficient), $\rho$ (the blood density) and $K_{R}$ (the friction parameter) are supposed to be constant. In particular, $K_{r}$ is related to the velocity profile assumed in the vessel. If a Poiseuille profile is assumed (which is of course a simplification), $K_{r}=8 \pi \nu$ (however, see [9] for other possible values). $A, P$ and $Q$ are the unknowns. By neglecting the acceleration in the independent ring model for the distension of the compliant vessel, we obtain the following algebraic model, often used in the medical bioengineering literature,

$$
P(A)=\frac{\beta}{A_{0}}\left(\sqrt{A}-\sqrt{A_{0}}\right),
$$

according to this pressure-area relationship, the wall displacement is proportional to the normal component of the applied external stress, where $A_{0}$ is the section area at rest and the coefficient $\beta$ supposed constant along 
the whole vessel, reads $\beta=\frac{\sqrt{\pi} h E}{\left(1-\sigma^{2}\right)}$. The constants $E, h$ and $\sigma$ are the Young modulus, the wall thickness and the Poisson ratio, respectively. We express the system $(1)$ in $(P, Q)$ variables:

$$
\left\{\begin{array}{l}
\partial_{t} P+\partial_{A} P \partial_{x} Q=0 \\
\partial_{t} Q+\partial_{x}\left(\alpha \frac{Q^{2}}{A}\right)+\frac{A}{\rho} \partial_{x} P=-K_{r} \frac{Q}{A} .
\end{array}\right.
$$

Using the pressure law (2), one has

The non-conservative form of (3) reads:

$$
\partial_{A} P=\frac{\partial P}{\partial A}=\frac{\beta}{2 A_{0} \sqrt{A}}
$$

$$
\partial_{t}\left(\begin{array}{l}
P \\
Q
\end{array}\right)+\left(\begin{array}{cc}
0 & \partial_{A} P \\
-\alpha u^{2} \partial_{P} A+\frac{A}{\rho} & 2 \alpha u
\end{array}\right) \partial_{x}\left(\begin{array}{l}
P \\
Q
\end{array}\right)=\left(\begin{array}{cc}
0 & 0 \\
0 & -\frac{K_{r}}{A}
\end{array}\right)\left(\begin{array}{l}
P \\
Q
\end{array}\right)
$$

where $u=Q / A$ is the mean velocity. Now, by linearizing the system (4) around a constant state $(A, u)=\left(A_{0}, 0\right)$ yields

$$
\left\{\begin{array}{l}
\partial_{t} P+\frac{\beta}{2 A_{0} \sqrt{A_{0}}} \partial_{x} Q=0, \\
\partial_{t} Q+\frac{A_{0}}{\rho} \partial_{x} P=-\frac{K_{r}}{A_{0}} Q .
\end{array}\right.
$$

Remark 2.1. As pointed out in [17], the choice of this linearization is reasonable as it can reproduce most of the essential features of the blood flow even when modelling the whole systemic tree.

If we set

$$
C^{\prime}=\frac{2 A_{0} \sqrt{A_{0}}}{\beta}, \quad L^{\prime}=\frac{\rho}{A_{0}}, \quad R^{\prime}=-\frac{\rho K_{r}}{A_{0}^{2}},
$$

then we can rewrite (5) in a simple linear form

$$
\left\{\begin{array}{l}
C^{\prime} \partial_{t} P+\partial_{x} Q=0 \\
L^{\prime} \partial_{t} Q+\partial_{x} P=-R^{\prime} Q
\end{array}\right.
$$

Note that the constants derived here coincide exactly with those of [9]. If we integrate this system in the $x$ direction on the interval $[0, l]$, we have

$$
\left\{\begin{array}{l}
C \frac{\mathrm{d} \hat{P}}{\mathrm{~d} t}+Q_{2}-Q_{1}=0 \\
L \frac{\mathrm{d} \hat{Q}}{\mathrm{~d} t}+P_{2}-P_{1}=-R \hat{Q}
\end{array}\right.
$$

Here, $\hat{P}=\frac{1}{l} \int_{0}^{l} P(x, t) \mathrm{d} x$ and $\hat{Q}=\frac{1}{l} \int_{0}^{l} Q(x, t) \mathrm{d} x$ stand for the mean pressure and flow-rate over the whole compartment, while

$$
Q_{1}(t)=Q(0, t), \quad P_{1}(t)=P(0, t), \quad Q_{2}(t)=Q(t, l), \quad P_{2}(t)=P(t, l),
$$

where $x=0$ is the upstream vessel boundary while $x=l$ is the downstream boundary. The new constants $R$, $L$ and $C$ read

$$
R=R^{\prime} l, \quad L=L^{\prime} l, \quad C=C^{\prime} l .
$$



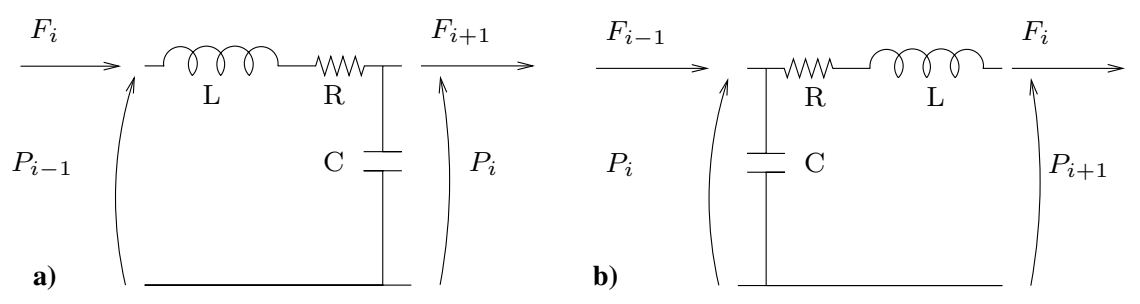

Figure 2. (a) the classical $\mathcal{L}$-circuit (left), (b) the inverse- $\mathcal{L}$-circuit.

Now assume that some upstream and downstream data are available. For instance suppose that $Q_{1}$ and $P_{2}$ are given. Then (8) represents a system of two equations and four unknowns. In order to close mathematically problem (8), we make the major assumption that

$$
\hat{P} \approx P_{1}, \quad \hat{Q} \approx Q_{2}
$$

relating the mean value over the vessel and the pointwise value at the inlet (or outlet) of the vessel. Then,

$$
C \frac{\mathrm{d} P_{1}}{\mathrm{~d} t}+Q_{2}-Q_{1}=0, \quad L \frac{\mathrm{d} Q_{2}}{\mathrm{~d} t}+P_{2}-P_{1}=-R Q_{2}, \quad t>0
$$

Remark 2.2. The waves are smooth and propagate very rapidly inside the cardiovascular system (between 1.5 and $10 \mathrm{~m} / \mathrm{s}$ ), the length of a single tube inside the systemic network can vary between few millimeters up to $10 \mathrm{~cm}$. Thus two pointwise values can be very close for a sufficiently small time delay, justifying why the averaged quantities are themselves close to the pointwise ones. This may explain why assumption (9) should be relevant from the physiological point of view.

Note that we have identified $\hat{P}$ with the upstream pressure and $\hat{Q}$ with the downstream flow-rate; what follows is coherent with this choice. However, the opposite choice would be acceptable as well, and would lead to a different circuit that we mention later (see Rem. 2.3).

In what follows we deal with the issue of "convergence" of 0D-models to 1D-models. To simplify our analysis, we will assume that the $1 \mathrm{D}$ system (7) holds for all $x \in \mathbb{R}$, and that it is replaced by an infinite number of elementary circuits like (10) whose length is $\Delta x$. More precisely, we connect an infinite number of similar elements

$$
C \frac{\mathrm{d} P_{i}}{\mathrm{~d} t}=-\left(Q_{i+1}-Q_{i}\right), \quad L \frac{\mathrm{d} Q_{i}}{\mathrm{~d} t}=-\left(P_{i}-P_{i-1}\right)-R Q_{i}
$$

where $\left(P_{i}, Q_{i}\right)$ indicate the pressure and the flow-rate computed at the point $x_{i}=i \Delta x$ for $i \in \mathbb{Z}$, (note that in the case of finite length, say for $i \in\{1, M\}$ this system would require $P_{0}$ to be provided at the inlet (beginning of the network) and $Q_{M+1}$ at the outlet of the network). System (11) can be equivalently regarded as the mathematical description of an electric circuit which is known as $\mathcal{L}$-circuit (see Fig. 2a). In fact, in this hydraulic/electric analogy, pressure and flow rate correspond to the electric voltage and current, the resistance $R$ is related to the blood viscosity, the inductance $L$ to the blood inertia and the capacitance $C$ to the wall compliance.

In order to establish our convergence result, we shall introduce a specific norm related to the nonlinear entropy function of system (1).

\subsection{From nonlinear entropy function to energy dissipated in the $\mathcal{L}$-circuit}

\subsubsection{From nonlinear entropy to an energy estimate}

One question arises after passing from the nonlinear model (3) to the linearized equations (7): is there some link between energetic considerations from both sides? The answer represent a new way to connect the entropy function related to instantaneous power dissipated in an electric circuit. 
Following [8], an a priori energy estimate for the nonlinear system (1) can be obtained. Define

$$
s(A, u)=\frac{\rho}{2} A u^{2}+\Psi
$$

where $\Psi=\Psi(A)$ is given by

$$
\Psi(A)=\int_{A_{0}}^{A} \psi\left(\zeta, A_{0}, \beta\right) \mathrm{d} \zeta
$$

and $\psi$ is a given wall law relating blood pressure and cross-section area. Here we consider a simple algebraic relation:

$$
p=\psi\left(A, A_{0}, \beta\right) \text {, with } \partial_{A} \psi>0 \text { and } \psi\left(A_{0}, A_{0}, \beta\right)=0
$$

for given constants $A_{0}$ and $\beta$. Then

$$
\Psi\left(A_{0}\right)=\Psi^{\prime}\left(A_{0}\right)=0, \text { and } \Psi^{\prime \prime}(A)>0, \quad \forall A>0 .
$$

Since $\Psi(A)$ is non-negative, so is $s(A, u)$. Thus we can define the averaged total energy of the $1 \mathrm{D}$ model by

$$
\mathcal{E}(t)=\int_{\mathbb{R}} s(A(x, t), u(x, t)) \mathrm{d} x, \quad \forall t>0 .
$$

We express $\Psi$, a function of the cross-section area, as a function of the pressure, namely:

$$
\Psi(A)=\int_{A_{0}}^{A} \psi(\zeta) \mathrm{d} \zeta=\int_{\psi\left(A_{0}\right)}^{\psi(A)} \psi \partial_{\psi} \zeta \mathrm{d} \psi=\Upsilon(P)
$$

$\zeta$ being the cross-section area variable associated with $\psi$. For the particular case of pressure law given by (2), $\zeta$ can be explicitly obtained as a function of $\psi$, namely $\zeta=\left[\frac{A_{0}}{\beta} \psi+\sqrt{A_{0}}\right]^{2}$. Consequently,

$$
\Upsilon(P)=\int_{0}^{P} \psi \partial_{\psi} \zeta \mathrm{d} \psi=2 \int_{0}^{P} \psi \frac{A_{0}}{\beta}\left[\frac{A_{0}}{\beta} \psi+\sqrt{A_{0}}\right] \mathrm{d} \psi=\frac{2}{3}\left(\frac{A_{0}}{\beta}\right)^{2} P^{3}+\frac{{\sqrt{A_{0}}}^{3}}{\beta} P^{2} \sim \frac{\left(\sqrt{A_{0}}\right)^{3}}{\beta} P^{2}=\frac{C^{\prime}}{2} P^{2} .
$$

The last approximation is due to the fact that the order of magnitude of the pressure inside the arterial tree is approximatively $P_{a} \sim 1200 \mathrm{~Pa}$ and the order of magnitude of $\beta$ is about $10^{5} \mathrm{~Pa} \cdot \mathrm{cm}$, then the cubic term is, in general, 10 to 100 times smaller than the the quadratic one; $C^{\prime}$ is the constant introduced in (6).

It remains to examine the first term on the r.h.s. of (12). Linearizing around the given cross-section area $A=A_{0}$, we have

Finally, starting from (12), that we rewrite in $P, Q$ variables,

$$
\frac{\rho}{2} A u^{2}=\frac{\rho}{2} \frac{Q^{2}}{A} \sim \frac{\rho}{2} \frac{Q^{2}}{A_{0}}=\frac{L^{\prime}}{2} Q^{2}
$$

$$
s(A, Q)=\frac{\rho}{2}\left(\frac{Q}{\frac{A_{0}}{\beta} P+\sqrt{A_{0}}}\right)^{2}+\frac{2}{3}\left(\frac{A_{0}}{\beta}\right)^{2} P^{3}+\frac{{\sqrt{A_{0}}}^{3}}{\beta} P^{2} \equiv e(P, Q),
$$

we obtain the following "linearized energy" functional

$$
e_{\operatorname{lin}}(P, Q)=\left(\frac{C^{\prime}}{2} P^{2}+\frac{L^{\prime}}{2} Q^{2}\right)
$$


that suggests the introduction of the following norm

$$
E(P, Q)(t)=\left(\int_{\mathbb{R}} e_{\operatorname{lin}}(P(x, t), Q(x, t)) \mathrm{d} x\right)^{\frac{1}{2}}=\left(\frac{C^{\prime}}{2}\|P\|_{L^{2}(\mathbb{R})}^{2}+\frac{L^{\prime}}{2}\|Q\|_{L^{2}(\mathbb{R})}^{2}\right)^{\frac{1}{2}},
$$

which is equivalent to the $L^{2}(\mathbb{R})$ norm. Multiplying the first equation of (7) by $P$, the second by $Q$, summing up the two equations and integrating over $\mathbb{R}$, we get:

Lemma 2.1. The solutions of system (7) are stable w.r. to norm E defined in (20); precisely

$$
\frac{\mathrm{d}}{\mathrm{d} t} E(P, Q)^{2}=-R \int_{\mathbb{R}} Q^{2}(x, t) \mathrm{d} x \leq 0, \quad \forall t \geq 0
$$

This proposition provides energy estimates for the linearized system as the nonlinear entropy function guarantees the stability for solutions of the nonlinear system (1) (see [8]).

\subsubsection{From dissipated power in the $\mathcal{L}$-circuit to a discrete energy estimate}

If we compute the instantaneous power dissipated in both branches of a single $\mathcal{L}$-circuit described by (11), we have [7]:

$$
\Pi_{i}=Q_{C, i} P_{C, i}+Q_{R L, i} P_{R L, i}
$$

where $\left(P_{C, i}, Q_{C, i}\right)$ are the values of pressure and flow-rate in the capacitor part, while $\left(P_{R L, i}, Q_{R L, i}\right)$ are those crossing the resistance $R$ and the inductance $L$. The pressure in the latter branch reads $P_{R L, i}=P_{i-1}-P_{i}$, while the pressure inside the capacitor is simply $P_{i}$. For the flow rate, by definition of a capacitor, we have

$$
Q_{C, i}=C \frac{\mathrm{d}}{\mathrm{d} t} P_{i}
$$

Summing (22) over $i$, and using the first equation of (11), one can write

$$
\sum_{i} \Pi_{i}=\sum_{i}-\left(Q_{i+1}-Q_{i}\right) P_{i}+Q_{i}\left(P_{i-1}-P_{i}\right)=0
$$

which is the consequence of Kirchhoff's laws. Re-expressing the latter equation one can write

$$
\sum_{i} \Pi_{i}=\frac{C}{2} \sum_{i} \frac{\mathrm{d}}{\mathrm{d} t} P_{i}^{2}+\frac{L}{2} \sum_{i} \frac{\mathrm{d}}{\mathrm{d} t} Q_{i}^{2}+\sum_{i} R Q_{i}^{2}=0
$$

Thus, we recover a stability estimate for the solutions of (11) in a discrete version of the norm (20), namely we can claim.

Lemma 2.2. Let us introduce the discrete energy

$$
E_{\Delta}\left(P_{\Delta}, Q_{\Delta}\right)=\left(\frac{C^{\prime}}{2} \sum_{i} \Delta x P_{i}^{2}+\frac{L^{\prime}}{2} \sum_{i} \Delta x Q_{i}^{2}\right)^{\frac{1}{2}}=\left(\frac{C^{\prime}}{2}\left\|P_{\Delta}\right\|_{l^{2}(\mathbb{R})}^{2}+\frac{L^{\prime}}{2}\left\|Q_{\Delta}\right\|_{l^{2}(\mathbb{R})}^{2}\right)^{\frac{1}{2}} .
$$

For the solution $\left(P_{\Delta}, Q_{\Delta}\right)$ of an infinite network of $\mathcal{L}$-circuits described by the semi-discrete scheme (11), the following discrete energy estimates holds

$$
\frac{\mathrm{d}}{\mathrm{d} t}\left(E_{\Delta}\left(P_{\Delta}, Q_{\Delta}\right)\right)^{2}=-R^{\prime}\left\|Q_{\Delta}\right\|_{l^{2}(\mathbb{R})}^{2} \leq 0
$$


In Section 2.1 we show how to build a hierarchy of models passing from 1D-nonlinear equations to the linear $1 \mathrm{D}$ system and ending with the $0 \mathrm{D}$ discretization. So it is possible to derive the corresponding hierarchy of energy estimates associated to each model. In what follows we use the previous norms to ensure the convergence of fully discrete schemes toward the solutions of the continuous system (7).

\subsection{Convergence of (11) to (7)}

If we turn back to the adimensionalized constants $R^{\prime} L^{\prime}$ and $C^{\prime}$, we can write (11) as

$$
C^{\prime} \frac{\mathrm{d} P_{i}}{\mathrm{~d} t}=-\frac{1}{\Delta x}\left(Q_{i+1}-Q_{i}\right), \quad L^{\prime} \frac{\mathrm{d} Q_{i}}{\mathrm{~d} t}=-R^{\prime} Q_{i}-\frac{1}{\Delta x}\left(P_{i}-P_{i-1}\right)
$$

where $\Delta x$ denotes now the (constant) length of the $i$ th element of the network. If we regard $Q_{i}$ as being the pointwise value at $x=x_{i}$ of the flow-rate function $Q(x, t)$ (that we assume regular enough), and the same assumption is made for $P_{i}$, then

$$
\left\{\begin{array}{l}
Q\left(x_{i+1}, t\right)=Q\left(x_{i}, t\right)+\Delta x \partial_{x} Q\left(x_{i}, t\right)+\frac{\Delta x^{2}}{2} \partial_{x x} Q\left(x_{i}, t\right)+O\left(\Delta x^{3}\right), \\
P\left(x_{i-1}, t\right)=P\left(x_{i}, t\right)-\Delta x \partial_{x} P\left(x_{i}, t\right)+\frac{\Delta x^{2}}{2} \partial_{x x} P\left(x_{i}, t\right)+O\left(\Delta x^{3}\right),
\end{array}\right.
$$

we draw that (25) can be regarded as a first order approximation in space of system (7). In this section, we prove that the scheme provided by the semi discrete system (25) is stable. Then, a linear scheme being stable and consistent, it is also convergent owing to the Lax-Richtmyer equivalence theorem (by Von Neumann analysis) $[21]$.

We discretize the system (25) in time using the first order forward Euler scheme,

$$
P_{i}^{n+1}=P_{i}^{n}-\frac{\lambda}{C^{\prime}}\left(Q_{i+1}^{n}-Q_{i}^{n}\right), \quad Q_{i}^{n+1}=\left(1-\frac{R^{\prime}}{L^{\prime}} \Delta t\right) Q_{i}^{n}-\frac{\lambda}{L^{\prime}}\left(P_{i}^{n}-P_{i-1}^{n}\right),
$$

where $P_{i}^{n}$ is an approximation of $P\left(x_{i}, t^{n}=n \Delta t\right.$ ) (and $Q_{i}^{n}$ of $Q\left(x_{i}, t^{n}=n \Delta t\right)$ ), $\Delta t$ being the time step, and $\lambda=\frac{\Delta t}{\Delta x}$ is the Courant number.

Theorem 2.1. The scheme (26) is unstable in the $L^{2}(\mathbb{R})$ norm under any hyperbolic CFL condition, i.e. if $\lambda \leq k$ for any choice of $k$ independent of $\Delta t$ and $\Delta x$. However, under the "parabolic CFL condition"

$$
\Delta t<\min \left(\frac{R^{\prime} C^{\prime} \Delta x^{2}}{2}, \frac{L^{\prime}}{R^{\prime}}\right)
$$

the scheme is stable for the $E_{\Delta}$ norm defined by (24). Moreover it provides solutions converging to the solution of system (7) w.r. to the $L^{2}(\mathbb{R})$ or $E$ norms.

Proof. Using the inverse Fourier transform formula we have

$$
P_{m}^{n}=\frac{1}{\sqrt{2 \pi}} \int_{-\frac{\pi}{\Delta x}}^{\frac{\pi}{\Delta x}} \mathrm{e}^{i m \Delta x \xi} \hat{P}^{n}(\xi) \mathrm{d} \xi, \quad Q_{m}^{n}=\frac{1}{\sqrt{2 \pi}} \int_{-\frac{\pi}{\Delta x}}^{\frac{\pi}{\Delta x}} \mathrm{e}^{i m \Delta x \xi} \hat{Q}^{n}(\xi) \mathrm{d} \xi,
$$

substituting this in (26) for $Q_{m+1}^{n}, Q_{m}^{n}, P_{m}^{n}, P_{m-1}^{n}$, we obtain

$$
\left\{\begin{array}{l}
P_{m}^{n+1}=\frac{1}{\sqrt{2 \pi}} \int_{-\frac{\pi}{\Delta x}}^{\frac{\pi}{\Delta x}} \mathrm{e}^{i m \Delta x \xi}\left[\hat{P}^{n}(\xi)-\frac{\lambda}{C^{\prime}}\left(\mathrm{e}^{i \Delta x \xi}-1\right) \hat{Q}^{n}(\xi)\right] \mathrm{d} \xi \\
Q_{m}^{n+1}=\frac{1}{\sqrt{2 \pi}} \int_{-\frac{\pi}{\Delta x}}^{\frac{\pi}{\Delta x}} \mathrm{e}^{i m \Delta x \xi}\left[\left(1-\frac{R^{\prime}}{L^{\prime}} \Delta t\right) \hat{Q}^{n}-\frac{\lambda}{L^{\prime}}\left(1-\mathrm{e}^{-i \Delta x \xi}\right) \hat{P}^{n}(\xi)\right] \mathrm{d} \xi
\end{array}\right.
$$


The Fourier transform being unique [21], we identify (29) and the formula (28) taken at the next time step $t^{n+1}$, to write:

$$
\left(\begin{array}{l}
\hat{P}^{n+1} \\
\hat{Q}^{n+1}
\end{array}\right)=\left(\begin{array}{cc}
1 & \frac{\lambda}{C^{\prime}} \bar{z} \\
-\frac{\lambda}{L^{\prime}} z & 1-\alpha
\end{array}\right)\left(\begin{array}{l}
\hat{P}^{n} \\
\hat{Q}^{n}
\end{array}\right)
$$

where we have set $\alpha=\frac{R^{\prime}}{L^{\prime}} \Delta t$ and $z=1-\mathrm{e}^{-i \Delta x \xi}$. We call $\mathcal{A}$ the amplification matrix in equation (30), and is function of the frequency $\xi$ and the constants $R^{\prime}, L^{\prime}, C^{\prime}$ and $\Delta t, \Delta x$.

In a first time, we estimate $\rho(\mathcal{A})$, the spectral radius of $\mathcal{A}$ in order to obtain a necessary condition of convergence.

Study of $\rho(\mathcal{A})$. The eigenvalues of $\mathcal{A}$ can be found by inspecting the roots of the characteristic polynomial

$$
\operatorname{det}(\mathcal{A}-\beta I)=\beta^{2}-(2-\alpha) \beta+\left(1-\alpha+\lambda^{2} \frac{|z|^{2}}{L^{\prime} C^{\prime}}\right)
$$

the associated discriminant is

$$
\Delta(\xi)=\alpha^{2}-4 \lambda^{2} \frac{|z|^{2}}{L^{\prime} C^{\prime}}, \quad \text { with }|z|^{2}=4 \sin ^{2}\left(\frac{\Delta x \xi}{2}\right) .
$$

The sign of $\Delta(\xi)$ as function of $\xi$

$\overline{\text { At first, we can remark that } \Delta(\xi)}$ is a monotone in $\left[0, \frac{\pi}{\Delta x}\right]$. We notice that $\Delta(0)=\alpha^{2}>0$. Then if $\Delta x<\frac{2}{R^{\prime}} \sqrt{\frac{L^{\prime}}{C^{\prime}}}$, one guarantees that $\Delta\left(\frac{\pi}{\Delta x}\right)<0$. This yields the existence of a unique root of $\Delta$ on $\left[0, \frac{\pi}{\Delta x}\right]$ which is

$$
\xi_{0}=\frac{2}{\Delta x} \arcsin \left(\frac{R^{\prime}}{4} \sqrt{\frac{C^{\prime}}{L^{\prime}}} \Delta x\right) .
$$

For $y$ small enough, $\arcsin (y)$ behaves like $y+\frac{1}{6} y^{3}+O\left(y^{5}\right)$ yielding

$$
\xi_{0}=\frac{R^{\prime}}{2} \sqrt{\frac{C^{\prime}}{L^{\prime}}}+\frac{R^{\prime 3} \Delta x^{2}}{3 \cdot 4^{3}}\left(\frac{C^{\prime}}{L^{\prime}}\right)^{\frac{3}{2}}+O\left(\Delta x^{4}\right)
$$

This means that $\xi_{0}$ belong to a small neighborhood of $\frac{R^{\prime}}{2} \sqrt{\frac{C^{\prime}}{L^{\prime}}}$ whose width depends on $\Delta x$.

As $\Delta(\xi)$ is monotone there are two subsets of $\left[-\frac{\pi}{\Delta x}, \frac{\pi}{\Delta x}\right]$, where its sign is constant. We call

$$
V_{\mathrm{ext}}\left(\xi_{0}\right)=\left[-\frac{\pi}{\Delta x},-\xi_{0}\right] \cup\left[\xi_{0}, \frac{\pi}{\Delta x}\right]
$$

the subset where $\Delta(\xi)$ is negative, while in $\left.V_{\mathrm{int}}\left(\xi_{0}\right)=\right]-\xi_{0}, \xi_{0}[, \Delta(\xi)$ is positive.

The case $\xi \in V_{\mathrm{ext}}\left(\xi_{0}\right)$

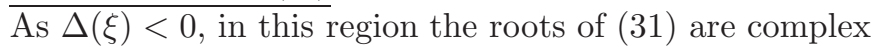

$$
\left|\beta_{ \pm}\right|^{2}=1-\alpha+\frac{\lambda^{2}|z|^{2}}{L^{\prime} C^{\prime}}
$$

1) Hyperbolic CFL condition: $\lambda$ is constant

If we take $\Delta x$ small enough so that $\frac{\pi}{2 \Delta x}$ belongs to the interior of $V_{\text {ext }}\left(\xi_{0}\right)$, one can write

$$
\left.\forall \xi \in] \frac{\pi}{2 \Delta x}+\frac{C^{\prime} R^{\prime}}{2 \lambda}, \frac{\pi}{\Delta x}\right], \quad \sin ^{2}\left(\frac{\Delta x \xi}{2}\right) \geq \frac{1}{2}+\frac{L^{\prime} C^{\prime}}{4 \lambda^{2}} \alpha .
$$


Note that $\frac{C^{\prime} R^{\prime}}{2 \lambda}$ is a constant, implying that there exists $\Delta x$ small enough such that $\frac{\pi}{2 \Delta x}+\frac{C^{\prime} R^{\prime}}{2 \lambda}<\frac{\pi}{\Delta x}$. Then (34) provides the lower bound for the eigenvalues:

$$
1+\frac{2 \lambda^{2}}{L^{\prime} C^{\prime}}<\left|\beta_{ \pm}(\xi)\right|=\rho(\mathcal{A}) .
$$

2) "Parabolic" CFL condition: $\Delta t<\left(R^{\prime} C^{\prime} \Delta x^{2} / 4\right)$

On the contrary, for this type of condition we have that

$$
\left|\beta_{ \pm}(\xi)\right| \leq 1-\alpha+\frac{4 \lambda^{2}}{L^{\prime} C^{\prime}}<1, \quad \forall \xi \in V_{\text {ext }}\left(\xi_{0}\right) .
$$

For the rest of the frequency spectrum, $\rho(\mathcal{A})$ is less or equal to one, for $\Delta t$ small enough.

The sufficient condition. Here we show the sufficient condition of stability under a "parabolic" CFL condition. The Parseval relation gives:

$$
\|P\|_{l^{2}}^{2}=\Delta x \sum_{i} P_{i}^{2}=\|\hat{P}\|_{L^{2}\left(-\frac{\pi}{\Delta x}, \frac{\pi}{\Delta x}\right)}^{2}=\int_{-\frac{\pi}{\Delta x}}^{\frac{\pi}{\Delta x}}|\hat{P}|^{2} \mathrm{~d} \xi
$$

thus we can write

$$
\left[E_{\Delta}(P, Q)\right]^{2}=\frac{C^{\prime}}{2}\|P\|_{l^{2}}^{2}+\frac{L^{\prime}}{2}\|Q\|_{l^{2}}^{2}=\frac{C^{\prime}}{2}\|\hat{P}\|_{L^{2}\left(-\frac{\pi}{\Delta x}, \frac{\pi}{\Delta x}\right)}^{2}+\frac{L^{\prime}}{2}\|\hat{Q}\|_{L^{2}\left(-\frac{\pi}{\Delta x}, \frac{\pi}{\Delta x}\right)}^{2} .
$$

We set $D=\operatorname{diag}\left(\sqrt{\frac{C}{2}}, \sqrt{\frac{L}{2}}\right), X=\left(\begin{array}{c}\hat{P} \\ \hat{Q}\end{array}\right)$, and we define the 2-norm of $X$ in $\mathbb{C}^{2}$ by $\|X\|_{2}=\sqrt{|\hat{P}|^{2}+|\hat{Q}|^{2}}$, then the previous energy term becomes

$$
\left[E_{\Delta}(P, Q)\right]^{2}=\int_{-\frac{\pi}{\Delta x}}^{\frac{\pi}{\Delta x}}\|D X(\xi)\|_{2}^{2} \mathrm{~d} \xi=\int_{-\frac{\pi}{\Delta x}}^{\frac{\pi}{\Delta x}}\|X(\xi)\|_{D}^{2} \mathrm{~d} \xi
$$

where $\|X\|_{D}=\|D X\|_{2}$. Applying matrix $D$ to (30) leads for a fixed $\xi$ to

$$
\left.D X^{n+1}(\xi)=D \mathcal{A}(\xi) D^{-1} D X^{n}(\xi), \quad \forall \xi \in\right] \frac{-\pi}{\Delta x} ; \frac{\pi}{\Delta x}[
$$

which gives the following estimate

$$
\left.\left\|X^{n+1}(\xi)\right\|_{D}^{2} \leq\left\|D \mathcal{A}(\xi) D^{-1}\right\|_{2}^{2}\left\|X^{n}(\xi)\right\|_{D}^{2}, \quad \forall \xi \in\right] \frac{-\pi}{\Delta x} ; \frac{\pi}{\Delta x}[.
$$

The quantity $\left\|D \mathcal{A} D^{-1}\right\|_{2}^{2}$ can be explicitly computed as

$$
\left\|D \mathcal{A} D^{-1}\right\|_{2}^{2}=\rho\left(\left(D \mathcal{A} D^{-1}\right)^{*} D \mathcal{A} D^{-1}\right) .
$$

The entries of the symmetric matrix $\mathcal{N}=\left(D \mathcal{A} D^{-1}\right)^{*} D \mathcal{A} D^{-1}$ are easy to obtain, and read

$$
\mathcal{N}=\left(\begin{array}{cc}
1+\frac{\lambda^{2}|z|^{2}}{L^{\prime} C^{\prime}} & -\alpha \frac{\lambda \bar{z}}{\sqrt{L^{\prime} C^{\prime}}} \\
-\alpha \frac{\lambda z}{\sqrt{L^{\prime} C^{\prime}}} & \frac{\lambda^{2}|z|^{2}}{L^{\prime} C^{\prime}}+(1-\alpha)^{2}
\end{array}\right)
$$


Its eigenvalues are

$$
\beta_{ \pm}=\frac{2+2 a+\Gamma \pm \sqrt{\Gamma^{2}+4 a \alpha}}{2}
$$

where $a=\frac{\lambda^{2}|z|^{2}}{L^{\prime} C^{\prime}}$ and $\Gamma=\alpha(\alpha-2)$. At this point, if we suppose that $\alpha<1$ and that $\frac{4 \lambda^{2}}{L^{\prime} C^{\prime}}<\alpha$, then for every $\xi, a<\alpha$ and

Finally, this gives the energy estimate:

$$
\beta_{ \pm}<\left(1+\frac{\sqrt{3}}{2} \alpha\right)^{2}
$$

$$
\begin{aligned}
E_{\Delta}\left(P^{n+1}, Q^{n+1}\right) & \leq(1+2 \sqrt{3} \alpha) E_{\Delta}\left(P^{n}, Q^{n}\right) \leq(1+2 \sqrt{3} \alpha)^{n} E_{\Delta}\left(P^{0}, Q^{0}\right) \\
& \leq \mathrm{e}^{\frac{\sqrt{3} R^{\prime}}{2 L^{\prime}}(n+1) \Delta t} E_{\Delta}\left(P^{0}, Q^{0}\right) \leq \mathrm{e}^{\frac{\sqrt{3} R^{\prime}}{2 L^{\prime}}(T+1)} E_{\Delta}\left(P^{0}, Q^{0}\right)
\end{aligned}
$$

where the bound is uniform in $\Delta t$ and $\Delta x$.

Instability under the hyperbolic CFL condition. To prove that the solution blows up in finite time for such a CFL condition, we take a particular solution. Let $v(\xi)$ be an eigenvector of $\mathcal{A}$ associated to the biggest eigenvalue $\beta_{+}$. Then

$$
v(\xi)=\left(\begin{array}{c}
\alpha+\sqrt{\Delta} \\
-\frac{2 \lambda z}{L^{\prime}}
\end{array}\right), \quad \beta_{+}=\frac{2-\alpha+\sqrt{\Delta}}{2}, \quad \Delta=\alpha^{2}-4 \frac{\lambda^{2}|z|^{2}}{L^{\prime} C^{\prime}} .
$$

We can provide a lower bound for $v(\xi)$ in the 2-norm as follows

$$
\|v(\xi)\|_{2}^{2}=\alpha^{2}+|\Delta|+4 \frac{\lambda^{2}|z|^{2}}{\left(L^{\prime}\right)^{2}} \geq 4 \frac{\lambda^{2}|z|^{2}}{\left(L^{\prime}\right)^{2}}, \quad \forall \xi \in V_{\mathrm{ext}}\left(\xi_{0}\right) .
$$

We call $\gamma=\frac{C^{\prime} R^{\prime}}{2 \lambda}$, and we set $I(\xi)=\mathbb{I}_{\left[\frac{\pi}{2 \Delta x}+\gamma, \frac{\pi}{\Delta x}\right]}(\xi)$, the characteristic function of $\left[\frac{\pi}{2 \Delta x}+\gamma, \frac{\pi}{\Delta x}\right]$. We set the Fourier transform of the initial condition to be

$$
X^{0}(\xi)=\left(\frac{L^{\prime}}{\lambda \sqrt{2}} \sqrt{\frac{\Delta x}{\frac{\pi}{2}-\gamma \Delta x}}\right) I(\xi) v(\xi), \quad \forall \xi \in\left[-\frac{\pi}{\Delta x}, \frac{\pi}{\Delta x}\right]
$$

For any fixed $\Delta x$, this function belongs to $L^{2}\left(-\frac{\pi}{\Delta x}, \frac{\pi}{\Delta x}\right)$. Applying (30) to this particular initial data gives the explicit solution as

$$
X^{n}=\beta_{+}^{n} X^{0} \text {. }
$$

Then, the following estimate holds

$$
\left\|X^{n}\right\|_{L^{2}(\mathbb{R})}^{2}=\int_{-\frac{\pi}{\Delta x}}^{\frac{\pi}{\Delta x}}\left|\beta_{+}\right|^{2 n}\left\|X^{0}\right\|_{2}^{2} \mathrm{~d} \xi=\frac{\left(L^{\prime}\right)^{2} \Delta x}{2 \lambda^{2}\left(\frac{\pi}{2}-\gamma \Delta x\right)} \int_{\frac{\pi}{2 \Delta x}+\gamma}^{\frac{\pi}{\Delta x}}\left|\beta_{+}\right|^{2 n}\|v(\xi)\|_{2}^{2} \mathrm{~d} \xi \geq\left(1+\frac{2 \lambda^{2}}{L^{\prime} C^{\prime}}\right)^{2 n} .
$$

For a hyperbolic CFL condition, the Courant number $\lambda$ is a fixed constant, and thanks to the previous inequality there is no possible bound for $X^{n}$ in $L^{2}\left(-\frac{\pi}{\Delta x}, \frac{\pi}{\Delta x}\right)$.

Remark 2.3. Another configuration of lumped circuit, called the inverse- $\mathcal{L}$ network, is driven by the following system of equations

$$
C^{\prime} \frac{\mathrm{d} P_{i}}{\mathrm{~d} t}=-\frac{1}{\Delta x}\left(Q_{i}-Q_{i-1}\right), \quad L^{\prime} \frac{\mathrm{d} Q_{i}}{\mathrm{~d} t}=-R^{\prime} Q_{i}-\frac{1}{\Delta x}\left(P_{i+1}-P_{i}\right)
$$


This is the symmetric form of the system (25), and is sketched by the electric network in Figure 2b. For this network, the Von Neumann analysis follows the same lines until one obtains the same discriminant (32) that guides the amplification matrix. Then it is clear that both networks can be regarded as approximations of the system (7).

\subsection{New 0D schemes for blood flow}

\subsubsection{The characteristic variables and the related diagonal system}

Through the change of variables

$$
\left(\begin{array}{l}
w \\
z
\end{array}\right)=\frac{1}{2}\left(\begin{array}{cc}
1 & \sqrt{\frac{L^{\prime}}{C^{\prime}}} \\
1 & -\sqrt{\frac{L^{\prime}}{C^{\prime}}}
\end{array}\right)\left(\begin{array}{l}
P \\
Q
\end{array}\right)
$$

the system (7) becomes

$$
\left\{\begin{array}{l}
\partial_{t} w+\frac{1}{\sqrt{C^{\prime} L^{\prime}}} \partial_{x} w=-\frac{R^{\prime}}{2 L^{\prime}}(w-z), \\
\partial_{t} z-\frac{1}{\sqrt{C^{\prime} L^{\prime}}} \partial_{x} z=\frac{R^{\prime}}{2 L^{\prime}}(w-z),
\end{array}\right.
$$

where $(w, z)$ are called the characteristic variables. This system is completely decoupled in the differential part, the two equations being coupled only by the source term.

The scheme (26) rewritten for the characteristic variables, becomes

$$
\left\{\begin{array}{l}
w_{i}^{n+1}=\left(1-\frac{R^{\prime}}{2 L^{\prime}} \Delta t\right) w_{i}^{n}-\frac{\lambda}{2 \sqrt{L^{\prime} C^{\prime}}}\left[w_{i+1}^{n}-w_{i-1}^{n}\right]+\frac{\lambda}{\sqrt{L^{\prime} C^{\prime}}}\left\{\frac{z_{i+1}^{n}-2 z_{i}^{n}+z_{i-1}^{n}}{2}\right\}+\frac{R^{\prime}}{2 L^{\prime}} \Delta t z_{i}^{n}, \\
z_{i}^{n+1}=\left(1-\frac{R^{\prime}}{2 L^{\prime}} \Delta t\right) z_{i}^{n}+\frac{\lambda}{2 \sqrt{L^{\prime} C^{\prime}}}\left[z_{i+1}^{n}-z_{i-1}^{n}\right]-\frac{\lambda}{\sqrt{L^{\prime} C^{\prime}}}\left\{\frac{w_{i+1}^{n}-2 w_{i}^{n}+w_{i-1}^{n}}{2}\right\}+\frac{R^{\prime}}{2 L^{\prime}} \Delta t w_{i}^{n} .
\end{array}\right.
$$

This corresponds to having discretized the characteristic system (37) by centered differences with the addition of two terms, one dissipative for the first equation, the other anti-dissipative for the latter one. This asymmetry indicates that the $\mathcal{L}$-circuit prioritizes backward waves and damps the amplitude of the forward ones. The centered differences are unstable under hyperbolic CFL conditions which might explain why we need a parabolic CFL condition to stabilize the numerical solutions. In the same way for an explicit discretization of (36), one has

$$
\left\{\begin{array}{l}
w_{i}^{n+1}=\left(1-\frac{R^{\prime}}{2 L^{\prime}} \Delta t\right) w_{i}^{n}-\frac{\lambda}{2 \sqrt{L^{\prime} C^{\prime}}}\left[w_{i+1}^{n}-w_{i-1}^{n}\right]-\frac{\lambda}{\sqrt{L^{\prime} C^{\prime}}}\left\{\frac{z_{i+1}^{n}-2 z_{i}^{n}+z_{i-1}^{n}}{2}\right\}+\frac{R^{\prime}}{2 L^{\prime}} \Delta t z_{i}^{n} \\
z_{i}^{n+1}=\left(1-\frac{R^{\prime}}{2 L^{\prime}} \Delta t\right) z_{i}^{n}+\frac{\lambda}{2 \sqrt{L^{\prime} C^{\prime}}}\left[z_{i+1}^{n}-z_{i-1}^{n}\right]+\frac{\lambda}{\sqrt{L^{\prime} C^{\prime}}}\left\{\frac{w_{i+1}^{n}-2 w_{i}^{n}+w_{i-1}^{n}}{2}\right\}+\frac{R^{\prime}}{2 L^{\prime}} \Delta t w_{i}^{n}
\end{array}\right.
$$

which is the complementary version of (38). Here again we see the opposite sign on the second order terms.

\subsubsection{Correctly upwinded $O D$ schemes}

Thanks to the previous arguments, we can list the major drawbacks of the standard electric circuits: expensive CFL, asymmetric wave propagation, no strict decrease of energy in the $E_{\Delta}$ norm (only a time exponential bound is available from (35)). To remove them, we propose here new schemes inspired by convenient hyperbolic discretizations of system (7). 
- First order discretization in space

A space discretization of system (7) by first order upwind schemes yields

$$
\left\{\begin{array}{l}
C \partial_{t} P_{i}=-\frac{1}{2}\left[Q_{i+1}-Q_{i-1}\right]+\frac{1}{2} \sqrt{\frac{C}{L}}\left(P_{i+1}-2 P_{i}+P_{i-1}\right) \\
L \partial_{t} Q_{i}=-\frac{1}{2}\left[P_{i+1}-P_{i-1}\right]+\frac{1}{2} \sqrt{\frac{L}{C}}\left(Q_{i+1}-2 Q_{i}+Q_{i-1}\right)-R Q_{i}
\end{array}\right.
$$

Its forward Euler time discretization gives the well-known Lax-Friedrichs' scheme. This scheme is strictly stable in the $E_{\Delta}$ norm, namely it's easy to prove the following result

Corollary 2.1. Under the CFL condition:

$$
\Delta t \leq \sqrt{L^{\prime} C^{\prime}} \Delta x=\sqrt{L C}
$$

the solution given by forward Euler time discretization of (40) satisfies the following energy estimate

$$
E_{\Delta}\left(P^{n+1}, Q^{n+1}\right) \leq E_{\Delta}\left(P^{n}, Q^{n}\right)
$$

The proof is straightforward, indeed in the characteristic variables the scheme reduces to the first order upwind scheme in both directions that can be set in a convex form. This ensures the decrease of $E_{\Delta}$ in time.

- A second order discretization in space can be used as well

For regular solutions it could be the Lax-Wendroff scheme:

$$
\left\{\begin{array}{l}
P_{i}^{n+1}=P_{i}^{n}-\frac{\Delta t}{2 C}\left[Q_{i+1}^{n}-Q_{i-1}^{n}\right]+\frac{\Delta t^{2}}{2 L C}\left(P_{i+1}^{n}-2 P_{i}^{n}+P_{i-1}^{n}\right) \\
Q_{i}^{n+1}=(1-\alpha) Q_{i}^{n}-\frac{\Delta t}{2 L}\left[P_{i+1}^{n}-P_{i-1}^{n}\right]+\frac{\Delta t^{2}}{2 L C}\left(Q_{i+1}^{n}-2 Q_{i}^{n}+Q_{i-1}^{n}\right) .
\end{array}\right.
$$

For less regular ones, a MUSCL scheme can be set up

$$
\left\{\begin{aligned}
Q_{i}^{n+1}= & (1-\alpha) Q_{i}^{n}-\frac{\Delta t}{2 L}\left[P_{i+1}^{n}-P_{i-1}^{n}\right]+\frac{\Delta t}{2 \sqrt{L C}}\left(Q_{i+1}^{n}-2 Q_{i}^{n}+Q_{i-1}^{n}\right) \\
& -\frac{1}{2}\left(1-\frac{\Delta t}{\sqrt{L C}}\right) \frac{\Delta t}{\sqrt{L C}} * \sqrt{\frac{C}{L}} *\left(\sigma_{1, i}^{n}-\sigma_{1, i-1}^{n}-\sigma_{2, i+1}^{n}+\sigma_{2, i}^{n}\right) \\
P_{i}^{n+1}= & P_{i}^{n}-\frac{\lambda}{2 C^{\prime}}\left[Q_{i+1}^{n}-Q_{i-1}^{n}\right]+\frac{\Delta t}{2 \sqrt{L C}}\left(P_{i+1}^{n}-2 P_{i}^{n}+P_{i-1}^{n}\right) \\
& -\frac{1}{2}\left(1-\frac{\Delta t}{\sqrt{L C}}\right) \frac{\Delta t}{\sqrt{L C}} *\left(\sigma_{1, i}^{n}-\sigma_{1, i-1}^{n}+\sigma_{2, i+1}^{n}-\sigma_{2, i}^{n}\right)
\end{aligned}\right.
$$

here $\alpha=\Delta t \frac{R}{L}$, and $\sigma_{1, i}^{n}=\min \bmod \left(w_{i+1}^{n}-w_{i}^{n}, w_{i}^{n}-w_{i-1}^{n}\right)$, and $\sigma_{2, i}^{n}=\min \bmod \left(z_{i+1}^{n}-z_{i}^{n}, z_{i}^{n}-z_{i-1}^{n}\right)$ are the slope limiters ensuring the $L^{1} \cap L^{\infty}$ stability of the scheme (see [10] for more details).

The major difference when discretizing system (7) with some upwind scheme relies on the way the variables are exchanged with neighbor elements: while the $\mathcal{L}$ network takes the pressure $P_{i-1}$ from the left neighbor cell and the flow rate $Q_{i+1}$ from the right one (see (11)), upwind schemes need both physical quantities on each side. 
TABLE 1. Error between the two energy functionals, OD solution against 1D linear solution and order of convergence.

\begin{tabular}{|c|c|c|c|}
\hline$\Delta x \mathrm{~cm}$ & $R_{\Delta}$ & $e_{\Delta x}$ & $\alpha$ \\
\hline 6.0000 & 0.0489 & 414.2032 & 1.0969 \\
\hline 1.2000 & 0.0416 & 70.8780 & 1.0998 \\
\hline 0.6000 & 0.0409 & 33.0700 & 1.1840 \\
\hline 0.3000 & 0.0405 & 14.5550 & 1.5399 \\
\hline 0.1200 & 0.0403 & 3.5500 & - \\
\hline
\end{tabular}

\subsection{Numerical assessment}

In this section, we perform a numerical simulation in order to check the above theoretical results. Using scheme (11), we compute the pressure $P_{0 \mathrm{D}}$, the flow rate $Q_{0 \mathrm{D}}$ inside a vessel of fixed length $(60 \mathrm{~cm})$, for different grid-size $\Delta x$.

For each $\Delta x$, we display the difference between the entropy functional $e$ from (18) and the linear energy $e_{\text {lin }}$ given in (19). Precisely, for each pair of solutions $\left(P_{0 \mathrm{D}}, Q_{0 \mathrm{D}}\right)$ we compute

$$
R_{\Delta}=\frac{\sum_{i, n}\left|e\left(P_{0 \mathrm{D}, i}^{n}, Q_{0 \mathrm{D}, i}^{n}\right)-e_{\mathrm{lin}}\left(P_{0 \mathrm{D}, i}^{n}, Q_{0 \mathrm{D}, i}^{n}\right)\right|}{\sum_{i, n} e\left(P_{0 \mathrm{D}, i}^{n}, Q_{0 \mathrm{D}, i}^{n}\right)}
$$

We show in Table 1, that this quantity is grid independent (which is expected because it is the discrete version of continuous functionals) and that the error is of only $4 \%$ approximatively. Then, we compare $\left(P_{0 \mathrm{D}}, Q_{0 \mathrm{D}}\right)$ to the numerical solution of (7) computed using the second order Lax-Wendroff scheme on a very refined grid (1000 grid-cells). In the third row of Table 1, using again the $E_{\Delta}$ norm, we compute

$$
e_{\Delta x}=\left(\Delta t \Delta x \sum_{n, i} e_{\mathrm{lin}}\left(P_{0 \mathrm{D}, i}^{n}-P_{e x}\left(x_{i}, t^{n}\right), Q_{0 \mathrm{D}, i}^{n}-Q_{e x}\left(x_{i}, t^{n}\right)\right)\right)^{\frac{1}{2}} .
$$

Then, the approximate order of convergence $\alpha_{\text {app }}$ is computed thanks to the standard formula [14]:

$$
\alpha_{\text {app }}=\frac{\log \left(e_{\Delta x_{1}} / e_{\Delta x_{2}}\right)}{\log \left(\Delta x_{1} / \Delta x_{2}\right)}
$$

and its value (see forth row in Tab. 1) corresponds actually to the first order exhibited when using the Taylor expansion. In Figure 3, we plot: the solution of the non-linear system (1) obtained using a second order TaylorGalerkin scheme [8], the linear solution used as reference in the previous table, and the solution $\left(P_{0 \mathrm{D}}, Q_{0 \mathrm{D}}\right)$ obtained for a grid size $\Delta x=1.2 \mathrm{~cm}$. We plot these solutions at four different locations in space $(x=0, x=20$, $x=40$ and $x=60 \mathrm{~cm}$ ). The time is given in seconds on the $x$-axis during two cycles. The pressure is imposed at the left boundary (see left upper corner of Fig. 3), and the flow-rate is given at the right outflow (right down corner in Fig. 3). We can observe that the difference between the solution of the linear system (7) and the 0D solution is negligible, while the differences are more important with the solution of the nonlinear system (1), however the non-linearity plays a role for high frequencies only. In Figure 4 we plot the values, over two cycles, of the discrete energy $E_{\Delta}$ (see (23)) and $\mathcal{E}_{\Delta}$ (the discrete version of $\mathcal{E}$ introduced in (15)) on the solution of (1). Differences are negligible, but they appear for higher values of pressure where the cubic term starts to account. We remark that entropy and energy do not decrease simply because the solutions is computed on a bounded interval with imposed boundary conditions at both ends of the interval. 

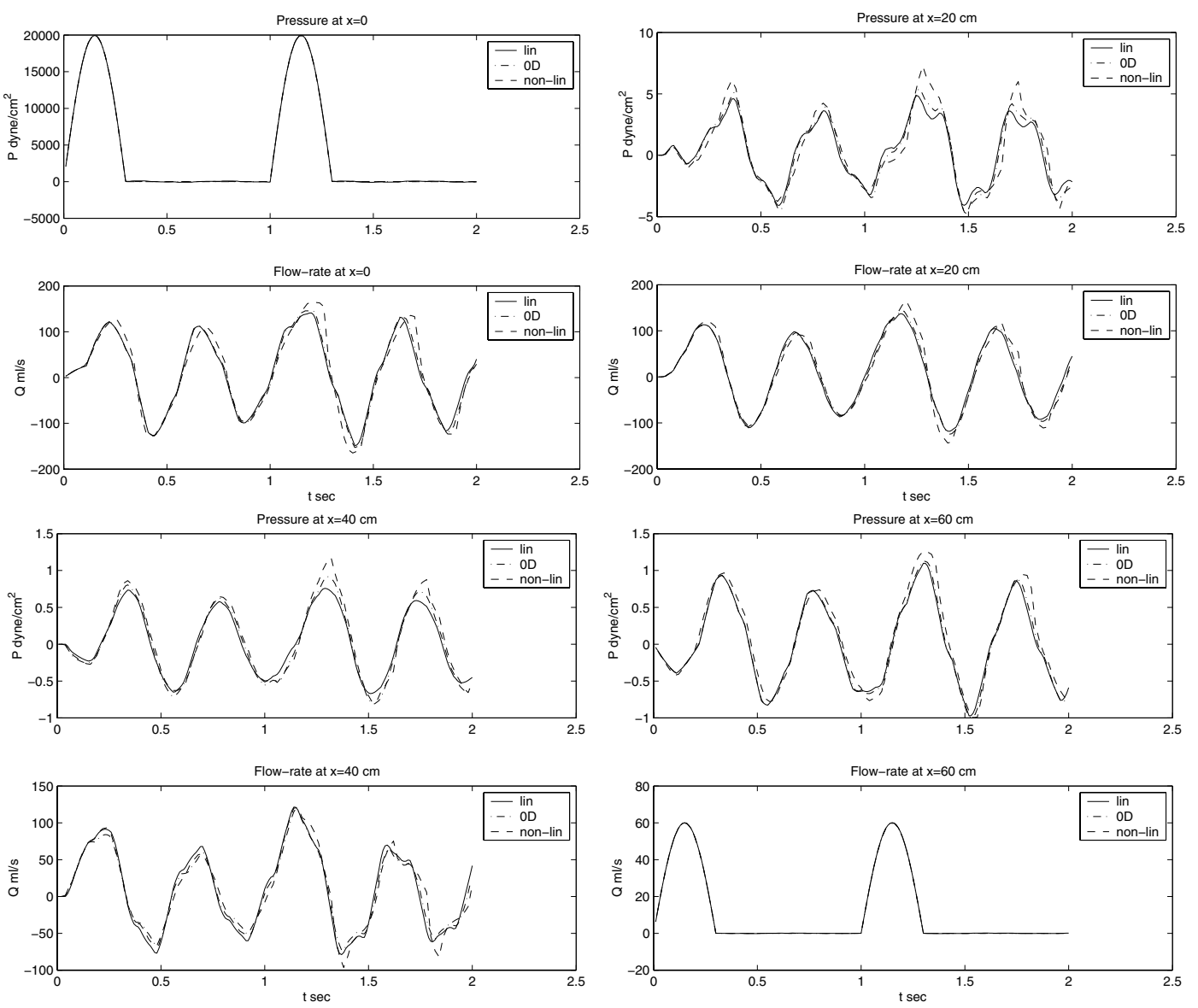

Figure 3. Display of numerical solutions at four different locations in space: linear 1D model, 0D network (50 elements), and nonlinear 1D model.

We consider a vessel whose length is $60 \mathrm{~cm}$, its radius $0.5 \mathrm{~cm}$, and its thickness $0.1 \mathrm{~cm}$. The blood viscosity is equal to $0.035 \mathrm{~cm}^{2} / \mathrm{s}$, its density is set to $1 \mathrm{~g} / \mathrm{cm}^{3}$, the Young modulus is set to $3 \times 10^{5} \mathrm{~Pa} \cdot \mathrm{cm}$, while the momentum-flux correction coefficient $\alpha$ is set to one (these data are taken from [9], p. 1.24).

\section{SMall Vessels}

The micro-circulation network formed by the arterioles, capillaries, as vessels with radii less than (roughly) $100 \mu \mathrm{m}$ is both topologically and functionally different from the network of large and medium size vessels [18]. At this spatial scale, blood can no longer be considered as a Newtonian fluid. The properties of the flow are strongly influenced by the individual red blood cells it contains in suspension. This affects fluid viscosity (known as Fahraeus effect), flow profiles and distribution of flow at bifurcations. Thus the equations used to model flow through the larger vessels are no longer valid. The large number of micro-circulation networks connecting each small artery to small vein also makes the method of discretely modelling individual vessel segments computationally prohibitive. To overcome this problems, a lumped parameter model of micro-circulation is used [19]. This model contains only a resistor and a capacitance. 


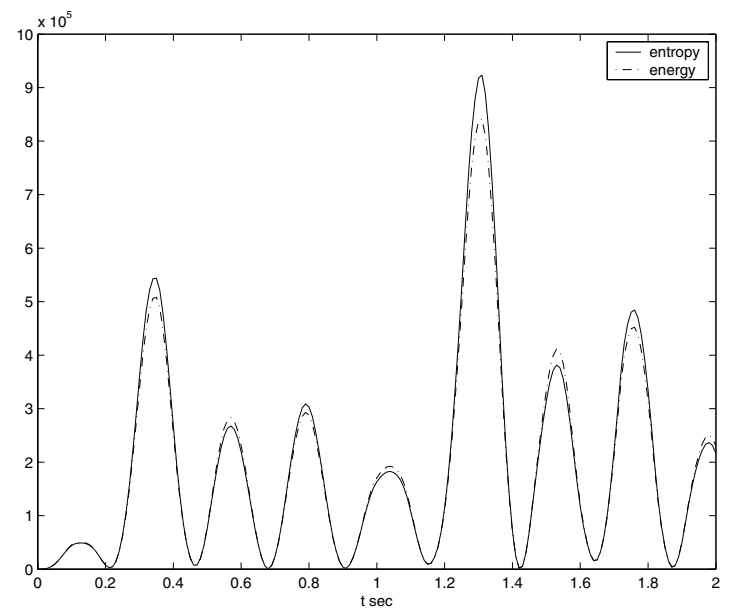

Figure 4 . Comparison between linear energy $E_{\Delta}$ and the entropy $\mathcal{E}_{\Delta}$ over two cycles of heart beat.

\subsection{Introducing the underlying equations}

In the small vessels it is reasonable to assume that the velocity profile is almost steady so that it solves the steady Stokes problem. If the vessel is axisymmetric, we can write the equation in cylindric coordinates

$$
\left\{\begin{array}{l}
\frac{1}{\rho} \partial_{x} P=\nu\left(\frac{1}{r} \partial_{r} V_{x}+\frac{\partial^{2} V_{x}}{\partial r^{2}}+\frac{\partial^{2}}{\partial x^{2}} V_{x}\right) \\
\frac{1}{\rho} \partial_{r} P=\nu\left(\partial_{r}\left(\frac{1}{r} \partial_{r}\left(r V_{r}\right)\right)+\frac{\partial^{2}}{\partial x^{2}} V_{r}\right)
\end{array}\right.
$$

and suppose the incompressibility condition

$$
\partial_{r} V_{r}+\frac{V_{r}}{r}+\partial_{x} V_{x}=0
$$

where $V_{r}$ and $V_{x}$ are the radial and the axial components of the velocity and $r$ is the radial coordinate. Following the asymptotic reduction already performed in [3], in a more general case, we suppose the following characteristic quantities: $V_{0}$ and $W_{0}$ are the characteristic radial and axial velocities, $X_{0}$ is the characteristic length, $R_{0}$ is the characteristic inner vessel radius, and the corresponding non-dimensional variables read

$$
r=R_{o} \tilde{r}, \quad x=X_{0} \tilde{x}, \quad t=\frac{X_{0}}{W_{0}} \tilde{t}, \quad V_{x}=W_{0} \tilde{V}_{\tilde{x}}, \quad V_{r}=V_{0} \tilde{V}_{\tilde{r}}, \quad P=\rho W_{0}^{2} \tilde{P} .
$$

We set $X_{0}=\frac{R_{0} W_{0}}{V_{0}}$ and $\epsilon=\frac{R_{0}}{X_{0}}$ is of order $\mathcal{O}\left(10^{-2}\right)$ in small vessels. The previous equations become

$$
\left\{\begin{array}{l}
\frac{\partial}{\partial \tilde{x}}(\tilde{r} \tilde{p})=\frac{\mu X_{0}}{W_{0} R_{0}^{2}}\left[\frac{\partial}{\partial \tilde{r}}\left(\tilde{r} \frac{\partial \tilde{V}_{\tilde{x}}}{\partial \tilde{r}}\right)\right] \\
\frac{\partial \tilde{p}}{\partial \tilde{r}}=0 \\
\frac{\partial}{\partial \tilde{r}}\left(\tilde{r} \tilde{V}_{\tilde{r}}\right)+\frac{\partial}{\partial \tilde{x}}\left(\tilde{r} \tilde{V}_{\tilde{x}}\right)=0
\end{array}\right.
$$


where we have neglected terms of order greater than 1 in $\epsilon$. The second equation in (44) implies that the pressure is constant on each cross-section, that's why we only introduce the averaged axial velocity

$$
\underline{\tilde{u}}=\frac{1}{\tilde{R}^{2}} \int_{0}^{\tilde{R}} 2 \tilde{V}_{\tilde{x}} \tilde{r} \mathrm{~d} \tilde{r}
$$

where $\tilde{R}$ is the inner vessel radius in the adimensional variables. Integrating the incompressibility equation in the radial direction and using the standard streamline condition:

$$
\frac{\mathrm{d} \tilde{R}}{\mathrm{~d} t}(x, t)=\frac{\partial \tilde{R}}{\partial \tilde{t}}+\tilde{V}_{\tilde{x}} \frac{\partial \tilde{R}}{\partial \tilde{x}}=\tilde{V}_{\tilde{r}},
$$

we obtain

$$
\frac{\partial}{\partial \tilde{t}} \tilde{R}^{2}+\frac{\partial}{\partial \tilde{x}}\left(\tilde{R}^{2} \underline{\tilde{u}}\right)=0 .
$$

In the same way, the first momentum equation becomes:

$$
\int_{0}^{\tilde{R}} \tilde{r} \partial_{\tilde{x}} P \mathrm{~d} r=\frac{\mu X_{0}}{W_{0} R_{0}^{2}} \tilde{R}\left[\partial_{\tilde{r}} \tilde{V}_{\tilde{x}}\right]_{\tilde{r}=\tilde{R}} .
$$

If we define the dimensional axial velocity by

$$
\underline{u}=\frac{1}{R^{2}} \int_{0}^{R} 2 r V_{x} \mathrm{~d} r
$$

we obtain that $\underline{u}=W_{0} \underline{\tilde{u}}$. This gives finally in the dimensional variables

$$
\left\{\begin{array}{l}
\partial_{t} A+\partial_{x} Q=0 \\
\frac{A}{\rho} \partial_{x} P=2 \pi \nu R\left[\partial_{r} V_{x}\right]_{r=R}
\end{array}\right.
$$

where the $A$ is the cross-section area of the vessel, and $Q=A \underline{u}$ is the flow-rate. To close the system we assume the typical velocity profile

$$
V_{x}=\frac{\gamma+2}{\gamma} \underline{u}\left[1-\left(\frac{r}{R}\right)^{\gamma}\right]
$$

(here this is very reasonable because the flow is almost steady see [23]). Notice that $\gamma=2$ corresponds to the Newtonian fluid. When $\gamma=9$ the profile is closed to the plug flow profile. Thanks to this assumption, the right hand-side of the latter equation in (45) becomes

$$
f=2 \pi \nu R\left[\partial_{r} V_{x}\right]_{r=R}=2 \nu R\left(-\frac{(\gamma+2)}{R} \underline{u}\right)=-2 \nu(\gamma+2) \underline{u}=-K_{r} \underline{u}
$$

leading to the following nonlinear system

$$
\left\{\begin{array}{l}
\partial_{t} A+\partial_{x} Q=0 \\
\frac{A}{\rho} \partial_{x} P=-K_{r} \frac{Q}{A}
\end{array}\right.
$$

Applying again the strategy used in Section 2.1, we express the latter system in the $P, Q$ variables using the closure law (2). Then we linearize around $A=A_{0}$ to obtain the following system

$$
\left\{\begin{array}{l}
C^{\prime} \partial_{t} P+\partial_{x} Q=0 \\
\partial_{x} P=-R^{\prime} Q
\end{array}\right.
$$




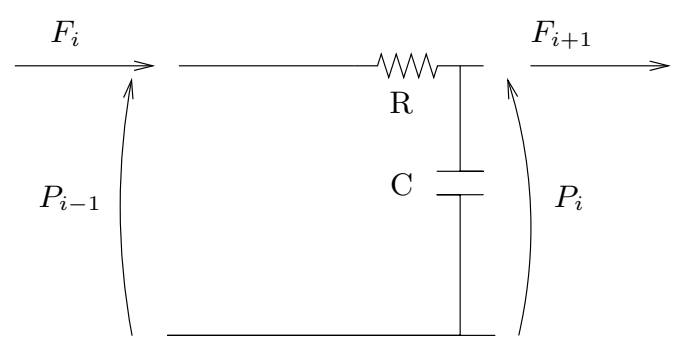

Figure 5. The $R C$-circuit.

that can be rewritten in a single equation that reads

$$
C^{\prime} \partial_{t} P-\frac{1}{R^{\prime}} \partial_{x x} P=0, \quad t \geq 0
$$

As previously the constants can be defined as in (6) by

$$
R^{\prime}=\frac{\rho K_{r}}{A_{0}^{2}}, \quad C^{\prime}=\frac{2 A_{0} \sqrt{A_{0}}}{\beta} .
$$

Remark 3.1. A similar linearized law is derived in $[4,5]$ without assumptions on the profile leading to different definitions of constants $R^{\prime}$ and $C^{\prime}$.

Remark 3.2. System (48) can be seen as the limit equation when the inductance term goes to 0 in (7), when the latter is written as a linear wave equation with a parabolic perturbation

$$
L^{\prime} C^{\prime} \partial_{t t} P+R^{\prime} C^{\prime} \partial_{t} P-\partial_{x x} P=0 \text {. }
$$

This shows that there is a coherent approach at every scale of approximation.

In a very standard way, we multiply equation (48) by $P$ and integrate by parts to obtain

$$
C^{\prime} R^{\prime} \frac{\mathrm{d}}{\mathrm{d} t} \int_{\mathbb{R}} P^{2} \mathrm{~d} x=-\int_{\mathbb{R}}\left|\partial_{x} P\right|^{2} \mathrm{~d} x \leq 0
$$

which means that the energy dissipated by this element is bounded, and depends only on the initial and boundary data. Note that the energy functional $E_{\Delta}$ introduced in 2.2 degenerates here to a single function of the pressure.

\subsection{Discretizing (48) with OD models}

As in Section 2.1 we integrate (47) in the axial direction and we suppose the same simplifying assumptions (9). This provides the following ODE system

$$
\left\{\begin{aligned}
C \frac{\mathrm{d} P_{i}}{\mathrm{~d} t} & =Q_{i}-Q_{i+1} \\
R Q_{i} & =P_{i-1}-P_{i},
\end{aligned}\right.
$$

that can be regarded again as the mathematical description of an $R C$-electric circuit like that depicted in Figure 5. Again, we have assumed that $R=R^{\prime} \Delta x$ and $C=C^{\prime} \Delta x$ where $\Delta x$ is the length of a single 
compartment described by (49). By letting the index $i$ vary from $-\infty$ to $+\infty$, and connecting together the same structure for all $i$, we obtain:

$$
C \frac{\mathrm{d} P_{i}}{\mathrm{~d} t}=Q_{i}-Q_{i+1}=\frac{1}{R}\left[P_{i+1}-2 P_{i}+P_{i-1}\right]
$$

which is a first order discretization (w.r. to $\Delta x$ ) of (48).

If we use the forward Euler scheme to discretize (50) in time, we need the following condition

$$
\Delta t \leq\left(\frac{R^{\prime} C^{\prime}}{2}\right) \Delta x^{2}
$$

in order to guarantee stability and therefore convergence toward the solution of the parabolic equation (48).

\section{Conclusions And Perspectives}

In this paper we have provided a rigorous approach for electric circuits whose physical constants $(R, L$ and $C)$ are parametrized on the actual length of the vessel in order to recover correct values for pressure or flow-rate.

For large and medium sized vessels we have shown the convergence of the lumped parameter model toward the solution of a 1D linear system, that is shown itself to be a linearization of the widely used 1D nonlinear system. Convergence is proven in a norm which derives from the entropy function of the 1D nonlinear system. Energy estimates were already available for the electric networks but no connection were made with 1D hyperbolic systems. Our result can therefore be regarded as a unifying approach for the three hierarchical models based on electric circuits, linear 1D hyperbolic systems and the (more complete) nonlinear 1D hyperbolic system.

This convergence result has prompted us to analyze from a new point of view the numerical features of the $R L C$-circuits, and underline their major drawbacks: stability of solutions under a "parabolic" CFL condition, the forward Euler discretization of $R L C$-circuits is bounded but not strictly decreasing (while the continuous problem and the semi-discrete scheme are actually strictly dissipative), the wave propagation is asymmetric. Moreover, this analysis led to propose new lumped parameter models inheriting properties of upwind schemes, that should be furtherly investigated.

The numerical tests have shown a good agreement between the linear energy and the entropy functional on solutions whose order of magnitude corresponds to physiological data. The convergence of the classical $R L C$ circuits was verified and a comparison between linear and nonlinear solutions of the 1D models was established.

In a second part of this paper, moving from the steady Stokes equations in an axisymmetric vessel, we have derived the whole hierarchy of equations for small vessels and showed that again $R C$-circuits converge toward a parabolic equation for the pressure.

The coupling of linear one-dimensional models with appropriate interface conditions is under investigation [13], based on the interface techniques necessary to consider couplings of systems of different kinds, namely between linear hyperbolic and parabolic PDE's, PDE's and ODE's in order to construct multiscale models. The final goal would be the treatment of the nonlinear systems that are currently used for blood flow simulations.

Acknowledgements. This study was partially supported by the RTN project HaeMOdel HPRN-CT-2002-00270 and the Bernoulli center at the École Polytechnique Fédérale de Lausanne during the special semester on the "Mathematical Modelling of the Cardiovascular System". The authors wish to express their thanks to Alessandro Veneziani and Luca Formaggia from M.O.X. of the Politecnico di Milano, for several fruitful discussions. The second author also acknowledges the contribution of the research projects MIUR-Cofin 2003 "Advanced Numerical Methods for Applications of Partial Differential Equations" and INDAM "Numerical Analysis for Scientific Computing and Advanced Applications", 2003. Finally, we would like to thank the reviewer for his useful comments and advice. 


\section{REFERENCES}

[1] A.P. Avolio, Multibranched model of the human arterial system. Med. Biol. Eng. Comput. 18 (1980) 709-119.

[2] B.S. Brook, S.A.E.G. Falle and T.J. Pedley, Numerical solutions for unsteady gravity-driven flows in collapsible tubes: evolution and roll-wave instability of a steady state. J. Fluid Mech. 396 (1999) 223-256.

[3] S. Čanić and E.H. Kim, Mathematical analysis of quasilinear effects in a hyperbolic model of blood flow through compliant axi-symmetric vessels. Math. Meth. Appl. Sci. 26 (2003) 1161-1186.

[4] S. Canić and A. Mikelić, Effective equations modeling the flow of a viscous incompressible fluid through a long elastic tube arising in the study of blood flow through small arteries. SIAM J. Appl. Dyn. Sys. 2 (2003) 431-463.

[5] A. Canić, D. Lamponi, S. Mikelić and J. Tambaca, Self-consistent effective equations modeling blood flow in medium-to-large compliant arteries. SIAM MMS (2004) (to appear).

[6] L. de Pater and J.W. van den Berg, An electrical analogue of the entire human circulatory system. Med. Electron. Biol. Engng. 2 (1964) 161-166.

[7] C.A. Desoer and E.S. Kuh, Basic Circuit Theory. McGraw-Hill (1969).

[8] L. Formaggia, J.F. Gerbeau, F. Nobile and A. Quarteroni, On the coupling of 3D and 1D Navier-Stokes equations for flow problems in compliant vessels. Comput. Methods Appl. Mech. Engrg. 191 (2001) 561-582.

[9] L. Formaggia and A. Veneziani, Reduced and multiscale models for the human cardiovascular system. Technical report, PoliMI, Milan (June 2003). Collection of two lecture notes given at the VKI Lecture Series 2003-07, Brussels 2003.

[10] E. Godlewski and P.-A. Raviart, Hyperbolic systems of conservation laws. Math. Appl., 3/4. Ellipses, Paris (1991).

[11] W.P. Mason, Electromechanical Transducers and Wave Filters (1942).

[12] F. Migliavacca, G. Pennati, G. Dubini, R. Fumero, R. Pietrabissa, G. Urcelay, E.L. Bove, T.Y. Hsia and M.R. de Leval, Modeling of the norwood circulation: effects of shunt size, vascular resistances, and heart rate. Am. J. Physiol. Heart Circ. Physiol. 280 (2001) H2076-H2086.

[13] V. Milišić and A. Quarteroni, Coupling between linear parabolic and hyperbolic systems of equations for blood flow simulations, in preparation.

[14] A. Quarteroni, R. Sacco and F. Saleri, Numerical Mathematics, 37 Texts Appl. Math. Springer-Verlag, New York (2000).

[15] V.C. Rideout and D.E. Dick, Difference-differential equations for fluid flow in distensible tubes. IEEE Trans. Biomed. Eng. BME-14 (1967) 171-177.

[16] P. Segers, F. Dubois, D. De Wachter and P. Verdonck, Role and relevancy of a cardiovascular simulator. J. Cardiovasc. Eng. 3 (1998) 48-56.

[17] S.J. Sherwin, V. Franke, J. Peiro and K. Parker, One-dimensional modelling of a vascular network in space-time variables. J. Engng. Math. 47 (2003) 217-250.

[18] N.P. Smith, A.J. Pullan and P.J. Hunter, An anatomically based model of transient coronary blood flow in the heart. SIAM J. Appl. Math. 62 (2001/02) 990-1018 (electronic).

[19] J.A. Spaan, J.D. Breuls and N.P. Laird, Diastolic-systolic coronary flow differences are caused by intramyocardial pump action in the anesthetized dog. Circ. Res. 49 (1981) 584-593.

[20] N. Stergiopulos, D.F. Young and T.R. Rogge, Computer simulation of arterial flow with applications to arterial and aortic stenoses. J. Biomech. 25 (1992) 1477-1488.

[21] J.C. Strikwerda, Finite difference schemes and partial differential equations. The Wadsworth \& Brooks/Cole Mathematics Series. Wadsworth \& Brooks/Cole Advanced Books \& Software, Pacific Grove, CA (1989).

[22] N. Westerhof, F. Bosman, C.J. De Vries and A. Noordergraaf, Analog studies of the human systemic arterial tree. J. Biomechanics 2 (1969) 121-143.

[23] F. White, Viscous Fluid Flow. McGraw-Hill (1986). 